\title{
Maximizing Communication Concurrency via Link-Layer Packet Salvaging in Mobile Ad Hoc Networks
}

Chansu Yu

Cleveland State University, c.yu91@csuohio.edu

Kang G. Shin

University of Michigan - Ann Arbor, kgshin@eecs.umich.edu

Lubo Song

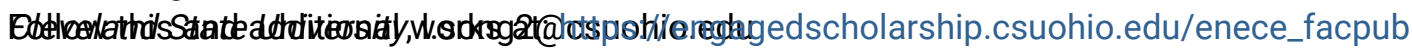

Part of the Digital Communications and Networking Commons

How does access to this work benefit you? Let us know!

\section{Publisher's Statement}

(C) 2007 IEEE. Personal use of this material is permitted. Permission from IEEE must be obtained for all other uses, in any current or future media, including reprinting/republishing this material for advertising or promotional purposes, creating new collective works, for resale or redistribution to servers or lists, or reuse of any copyrighted component of this work in other works.

\section{Original Citation}

Yu, C., Shin, K. G., \& Song, L. (April 01, 2007). Maximizing communication concurrency via link-layer packet salvaging in mobile ad hoc networks. IEEE Transactions on Mobile Computing, 6, 4, 449-462.

\section{Repository Citation}

Yu, Chansu; Shin, Kang G.; and Song, Lubo, "Maximizing Communication Concurrency via Link-Layer Packet Salvaging in Mobile Ad Hoc Networks" (2007). Electrical Engineering \& Computer Science Faculty Publications. 96. https://engagedscholarship.csuohio.edu/enece_facpub/96

This Article is brought to you for free and open access by the Electrical Engineering \& Computer Science Department at EngagedScholarship@CSU. It has been accepted for inclusion in Electrical Engineering \& Computer Science Faculty Publications by an authorized administrator of EngagedScholarship@CSU. For more information, please contact library.es@csuohio.edu. 


\title{
Maximizing Communication Concurrency via Link-Layer Packet Salvaging in Mobile Ad Hoc Networks
}

\author{
Chansu Yu, Member, IEEE, Kang G. Shin, Fellow, IEEE, and Lubo Song
}

\begin{abstract}
Carrier-sense Medium Access Control (MAC) protocols such as the IEEE 802.11 Distributed Coordination Function (DCF) avoid collisions by holding up pending packet transmission requests when a carrier signal is observed above a certain threshold. However, this often results in unnecessarily conservative communication, thus making it difficult to maximize the utilization of the spatial spectral resource. This paper shows that a higher aggregate throughput can be achieved by allowing more concurrent communications and adjusting the communication distance on the fly, which needs provisions for the following two areas: On the one hand, carrier sense-based MAC protocols do not allow aggressive communication attempts when they are within the carrier senseable area. On the other hand, the communication distance is generally neither short nor adjustable because multihop routing protocols strive for providing minimum hop paths. This paper proposes a new MAC algorithm, called Multiple Access with Salvation Army $(M A S A)$, which adopts less sensitive carrier sensing to promote more concurrent communications and adjusts the communication distance adaptively via "packet salvaging" at the MAC layer. Extensive simulation based on the ns-2 has shown MASA to outperform the DCF, particularly in terms of packet delay. We also discuss the implementation of MASA based on the DCF specification.
\end{abstract}

Index Terms-Mobile ad hoc networks, carrier sense, medium access control, capture effect, nondeterministic algorithm.

\section{INTRODUCTION}

$\mathrm{P}$ ATH loss in wireless communication fundamentally limits the performance of mobile ad hoc networks by requiring intermediate relay nodes to participate in delivery of data packets, but it creates a new opportunity for distant nodes in the network to reuse the shared radio channel simultaneously. However, this increases aggregate cochannel interference, rendering it important to make sure each data transfer "survives" in the presence of interference.

Carrier sense (CS)-based medium access control (MAC) algorithms alleviate the interference problem by mandating a node to hold up pending transmission requests when it observes a carrier signal above a CS threshold [2]. A lower CS threshold will result in less interference and, hence, a better signal-to-interference-ratio (SIR) at the receiver. However, it may have a negative impact on network capacity because it allows fewer concurrent data transfers in the network. Therefore, the CS threshold should be configured to balance between the spatial reusability and the interference problem [3]. Two important factors in this regard are communication distance [4] and capture effect [5]. Consider a land mobile radio environment where the signal strength attenuates as the fourth power of the distance. Halving the communication distance results in a 16 times stronger signal at the

- C. Yu and L. Song are with the Department of Electrical and Computer Engineering, Cleveland State University, 2121 Euclid Avenue, SH437, Cleveland, OH 44115. E-mail: \{c.yu91, l.song2\}@csuohio.edu.

- K.G. Shin is with the Department of Electrical Engineering and Computer Science, University of Michigan, Ann Arbor, MI 48109-2122.

E-mail:kgshin@eecs.umich.edu. receiver, meaning much more robust communication to interference. In other words, if communication distance is short, a low CS threshold would be an overkill because the SIR is high enough anyway.

Earlier, we proposed a nondeterministic MAC algorithm, called the Multiple Access with Salvation Army (MASA) [1], that adopts a higher CS threshold to encourage more spatial reuse but adjusts the communication distance on the fly by salvaging packets at the MAC layer to mitigate the interference problem. A key idea is that, even if an intended receiver could not receive a data packet due to interference, a third party node among those in between the sender and the receiver, called the salvation army, "captures" or "salvages" the packet and makes progress toward the receiver. While packet salvaging is not new at the network layer [6], [7], MASA operates at the MAC layer for faster salvaging. It is also different from other nondeterministic MAC layer schemes [8], [9], [10], [11], [12] in the sense that 1 ) it is purely a MAC-layer scheme, which still has its clear advantages over cross-layer design discussed in the literature, and 2) it uses a deterministic routing path whenever possible and salvages packets only when the primary path breaks.

This paper extends our earlier work on MASA by significantly expanding its evaluation, particularly in the following two areas:

- To evaluate the efficiency of MASA, it is important to understand how many packets are actually salvaged and how many of them are successfully forwarded to the original receivers. In fact, this evaluation helped us improve the MASA algorithm as described in Section 4.2. For the simulation 
parameters we have used, the results show that about 30 percent of packets are salvaged and more than 80 percent of those salvaged are successfully forwarded.

- We investigated how MASA performs in the presence of unreliable links. Unlike most simulation scenarios used in the literature, a real-world scenario is often characterized by the high degree of link unreliability as recently observed in [13], [14], [15]. We use the shadowing propagation model [16] and found that MASA salvages more when link unreliability is higher.

The rest of the paper is structured as follows: Section 2 presents the system model, including the DCF and the radio propagation model that determines packet capturing. Section 3 analyzes the maximum network throughput in terms of CS threshold and communication distance. The proposed packet-salvaging MAC algorithm, called MASA, is presented in Section 4. Extensive simulation based on ns-2 [16] has been conducted to evaluate various performance metrics, such as packet delay, packet delivery ratio, routing control overhead, and packet queuing requirement, which are reported in Section 5. Section 6 overviews related work including previous packet-salvaging schemes and other throughput-enhancing techniques, such as transmit power control (TPC) and transmit rate control (TRC). Section 7 draws conclusions and describes future directions of this study.

\section{System Model}

As discussed in Section 1, carrier sensing is used to avoid unwanted interferences, but it potentially limits the spatial channel utilization in wireless ad hoc networks. This section discusses the radio-propagation model and the DCF with a special focus on its spatial reusability.

\subsection{Radio-Propagation Model}

Radio propagation within a mobile channel is described by means of three effects: attenuation due to distance $(d)$ between the sender (node $i$ ) and the receiver (node $j$ ), shadowing due to the lack of visibility between the two nodes, and fading due to multipath propagation [5]. This paper assumes a simple propagation model by considering only the path loss due to communication distance. According to the two-ray ground propagation model, the mean received signal power $\left(P_{r}\right) \leftarrow$ follows an inverse distance power-loss law, where an exponent $\alpha \psi$ assumes values between 2 and 4 , and is typically 4 in land mobile radio environments [5]. In other words, $P_{r \psi}=P_{t, i} \gamma_{i j}$, where $P_{t, i}$ is the radio transmit power of node $i \psi$ and $\gamma_{i j \psi} \propto d^{-\alpha \psi}$ is the channel gain from node $i \psi$ to node $j$. In the $915 \mathrm{MHz}$ WaveLAN radio hardware, the transmit power is $24.5 \mathrm{dBm}$ and the receive sensitivity is $-72 \mathrm{dBm}$, which is translated to a $250 \mathrm{~m}$ or shorter distance between the sender and the receiver $(d)$ for successful communication [17], [18].

When another node (say, node $k$ ) in node $j$ 's proximity attempts to transmit during the communication between node $i$ yand $j$, it may cause collision at the receiver (node $j$ ).

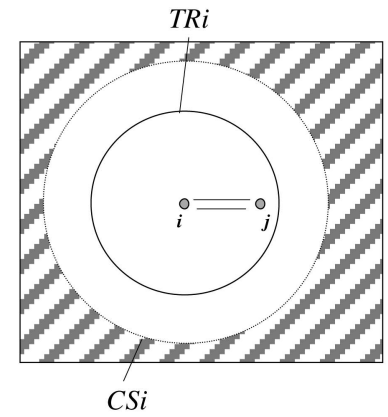

(a)

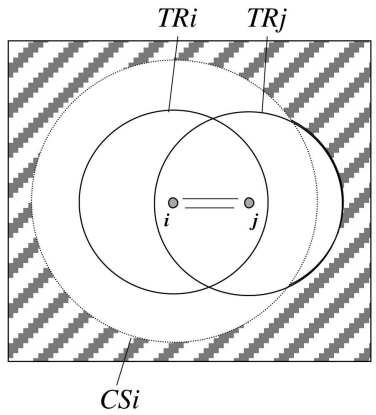

(b)
Fig. 1. Potential vulnerable space (hatched area) in the DCF. (a) With CS. (b) With CS and RTS/CTS.

However, collision does not necessarily destroy all packets involved and one of them may survive if the received signal power is far greater than that of the interfering signal. This is one of the key features in a mobile radio environment known as capture effect [5]. In general, in order for node $j$ to receive a signal from node $i \psi$ correctly, the instantaneous signal to noise ratio must be larger than a certain threshold, called capture ratio or $z_{0}$, which is determined by the sensitivity and capability of the radio receiver circuitry, i.e.,

$$
S I R \psi \stackrel{P_{t, i} \gamma_{i j}}{N_{0}+\sum_{k \neq i} P_{t, k} \gamma_{k j \psi}}>z_{0}, \psi
$$

where $N_{0}$ is the background nbise power. $z_{0}$ ranges from 1 (perfect capture) to $\infty$ (no capture) [5].

\subsection{DCF (IEEE 802.11 MAC)}

A MAC protocol for multiaccess media is essentially a distributed scheduling algorithm that allocates the available spectral resource to requesting nodes. In general, the performance of a MAC protocol is greatly affected by collisions because a packet transmission to a busy receiver is not queued but incurs transmission failures for both packets. For example, a simple algorithm such as $A L O H A$ allows many data transfers to occur simultaneously but its throughput is critically limited because of the lack of collision avoidance mechanism. In order for a sender to transmit a packet successfully, other interfering nodes within a receiver's reception area should not attempt to transmit during the sender's transmission. They are called vulnerable space (VS) [19] and vulnerable period [20], respectively. Carrier-sensing protocols shrink the VS by suppressing the neighboring nodes of the transmitter. On the other hand, the spatial area, which could have been used for other communications but is wasted due to excessive CS, is called wasted space (WS) in this paper. A MAC algorithm should reduce VS but, at the same time, reduce WS. We will discuss below how VS and WS are related to CS threshold, receive sensitivity, and capture ratio. They are directly translated to CS zone, transmission zone, and capture $(C P)$ zone, respectively, which will be detailed later in this and the following section.

When node $i$ transmits a data packet to node $j \psi$ as in Fig. 1a, the CS zone of the sender (node $i$ ), denoted as $C S_{i}$, 


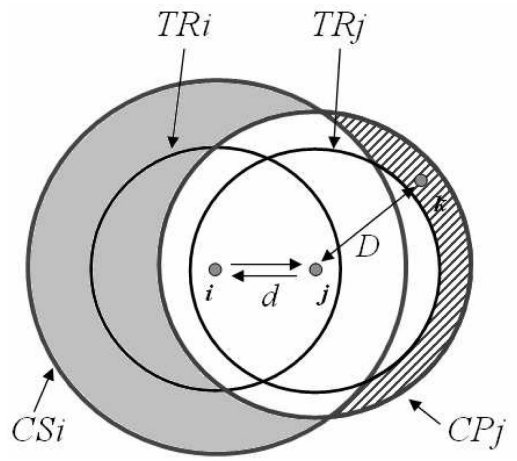

(a)

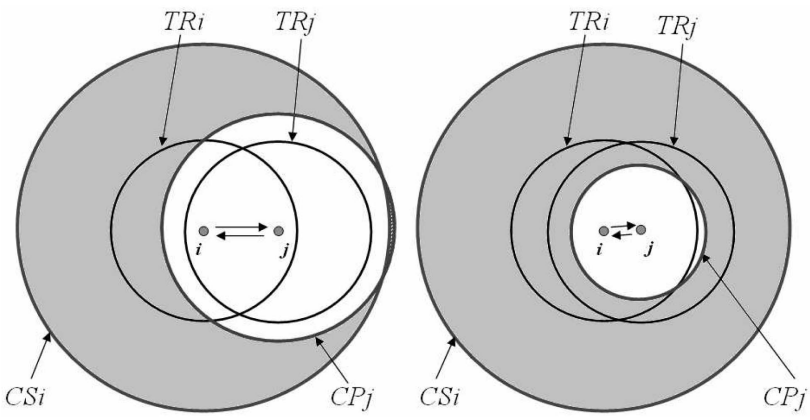

(b)

Fig. 2. Vulnerable space (VS) and wasted space (WS). (In (a), VS is the hatched area on the right and WS is the shaded area on the left. In (b), VS is empty.) (a) Relationship with CS, CP, and TR zones. (b) $d=200 \mathrm{~m}$ and $100 \mathrm{~m}\left(z_{0}=10 \mathrm{~dB}, \alpha \psi 4\right)$.

is a circular region in which a node would observe the sender's signal to be higher than the CS threshold. Since nodes in the CS zone would not cause any trouble, $V S \Psi \overline{C S_{i}}$. The DCF optionally employs the RTS/CTS (Request-to-Send and Clear-to-Send) handshake to further reduce collisions. By overhearing two short control packets, every neighboring node of two communicating nodes $i$ and $j \psi$ recognizes their communication and refrains from initiating its own transmission. This is known as virtual carrier sensing (VCS) [21] and the potential VS is now reduced to $\overline{C S_{i \psi} \cup T R_{j} \psi}$ as shown in Fig. 1b. Here, $T R_{j \psi}$ is the transmission zone of node $j$, i.e., any node in $T R_{j \psi} \mathrm{can}$ receive node $j^{\prime}$ s transmission, such as CTS.

The DCF works reasonably well in one-hop infrastructure networks. However, in wireless multihop networks, the carrier sensing and the RTS/CTS handshake creates WS, better known as the exposed terminal problem [22]. It may cause a live link to be considered broken when an intended receiver is unnecessarily exposed to another pair's communication and, thus, cannot respond to RTS. In summary, the DCF is effective in combating collisions (VS), but it may have a negative impact on performance by reserving unnecessarily large space (WS).

\section{Throughput Analysis of Multihop Network}

In order to provide a feel for the effect of communication distance and carrier sensing, this section presents the throughput analysis of multihop networks. Analyses without considering the effect of carrier sensing can be found in [4], [23], [24]. Recently, Zhu et al. extended the analysis to find the optimal CS threshold that maximizes the spatial utilization [25]. Yang and Vaidya extended it further by including the MAC overhead [26]. Xu et al. [27] analyzed the effectiveness of VCS in terms of communication distance and CS threshold. Our analysis is different from theirs in that it accounts for the effect of multiple interferers as well as communication distance and CS threshold. Section 3.1 considers the case with a single interferer while Section 3.2 provides the maximum throughput analysis with multiple interferers.

\subsection{VS and WS Analysis with a Single Interferer}

Assuming that $N_{0}$ is ignorable and the transmit power is constant, (1), for a single interfering node $k$, becomes

$$
\operatorname{SIR} \nLeftarrow \frac{P_{t, i} \gamma_{i j \psi}}{P_{t, k} \gamma_{k j \psi}}=\frac{\gamma_{i j \psi}}{\gamma_{k j \psi}}=\frac{d^{-\alpha \psi}}{D^{-\alpha}}=\left(\frac{D}{d}\right)^{\alpha}>z_{0} \text { or } D>z_{0}^{1 / \alpha \Downarrow}, \psi(2) \leftarrow
$$

where $d \psi$ and $D \psi$ denote the sender-to-receiver $(i-j)$ and interferer-to-receiver $(k-j)$ distance, respectively, as shown in Fig. 2a. Equation (2) defines the capture zone of node $j \psi$ denoted as $C P_{j}$. Any node outside of $C P_{j \psi}$ does not cause collisions to the communication between nodes $i$ and $j$ due to the capture effect. It means that $V S \& C P_{j} \cap \overline{\left(C S_{i \psi} \cup T R_{j}\right)}$, which is marked as the hatched area on the right in Fig. 2a. On the other hand, since the capture zone is the area that needs to be protected, the carrier sensing and the RTS/CTS handshake in fact protect a larger space than needed. That is, $W S \notin\left(C S_{i \psi} \cup T R_{j}\right)-C P_{j}$, which is marked as the shaded area on the left in Fig. 2a. Collisions are not entirely avoidable because VS is not empty. However, the large WS is a more serious problem.

In our baseline model, $z_{0}=10$ (or $10 \mathrm{~dB}$ ), $\alpha \psi 4$, and the maximum transmit distance is $250 \mathrm{~m}$. Fig. $2 \mathrm{~b}$ shows two cases when $d=200 \mathrm{~m}$ and $100 \mathrm{~m}$, respectively. Note that VS becomes negligible but a large WS could pose a serious performance problem in the two figures of Fig. 2b. Note also that $C S_{i \Downarrow} \supset T R_{j}$, meaning that RTS/CTS handshake is not useful in reducing collisions, as observed in [28], [29]. The second figure of Fig. $2 \mathrm{~b}$ shows that the problem is even more significant with a short communication distance $(d=100 \mathrm{~m})$. The sender-receiver pair becomes more robust to interference, i.e., $C P_{j}$ becomes smaller and it results in an even larger WS. A straightforward solution to the large WS and the corresponding exposed terminal problem is to make $C S_{i \psi}$ small (or, equivalently, to increase the CS threshold). However, when dis not small, it may increase VS and cause collisions. Therefore, it is imperative to have an adaptive capability that adjusts the CS threshold or the communication distance depending on the local network condition.

\subsection{Maximum Throughput with Multiple Interferers}

Generally, there could be more than one interferer or, equivalently, more than one sender. The maximum total end-to-end throughput, $T_{e}$, is attained when the number of 


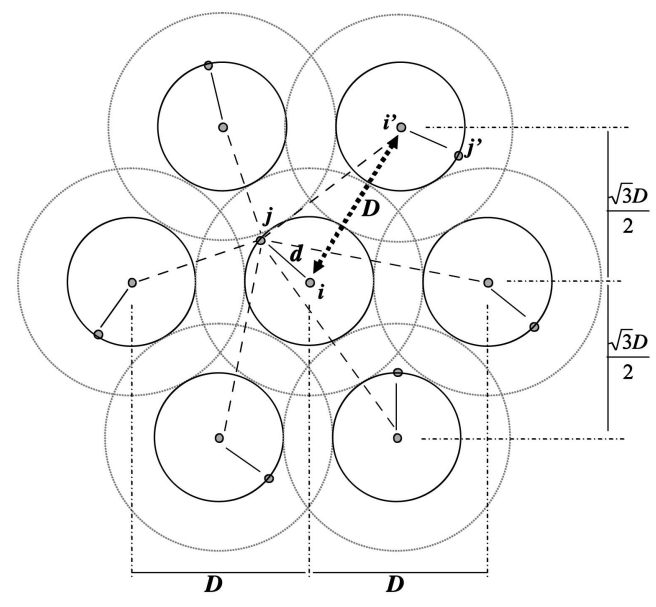

Fig. 3. Constellation of senders for maximum throughput.

senders that can simultaneously transfer data is maximized. Multiplying this number by the wireless link bandwidth and then dividing by the average number of hops between the source and the destination will yield an estimate of $T_{e}$. In the following analysis, we assume a heavily loaded network in which each node is always backlogged and has a packet to transmit whenever it is allowed. Perfect MAClayer coordination is assumed without collision so that spatial channel utilization is maximized as similarly assumed in [26].

The number of senders can be maximized when they are located as close to each other as possible without interfering with each other's data transfer. This is similar to the cochannel interference problem in cellular networks [22]. Consider the constellation of senders as in Fig. 3, which is the densest arrangement of senders. Assuming that each communication distance is $d$, we want to find the sender-to-sender distance $D \psi$ that allows all data transfers to be simultaneously successful. We only consider the six first-tier interferers because the interference from them is much stronger than that from second-tier interferers and beyond. Now, the worst-case interference to the communication between nodes $i \psi$ and $j \psi$ happens when the six interferers are $(D-d),(D-d),(D-d / 2), D,(D+d / 2)$, and $(D+d)$ apart from the receiver $j$, respectively [26]. Therefore, using (1) and (2),

$S I R \psi \leftarrow$

$\frac{d^{-\alpha \psi}}{2(D-d)^{-\alpha}+(D-d / 2)^{-\alpha}+D^{-\alpha}+(D+d / 2) \stackrel{-\alpha}{\longleftarrow}+(D+d) \stackrel{-\alpha}{\longleftarrow}}>z_{0}$.

If $D_{\min }$ is the minimum $D$ that satisfies (3), the maximum number of senders in an $L \times L$ isquare network area is

$$
\frac{L}{D_{\min }} \times \frac{L \psi}{\frac{\sqrt{3}}{2} D_{\min }}=\frac{2 L^{2}}{\sqrt{3} \not \phi_{\min }^{2}} \cdot \psi
$$

Since the average distance between a source-destination pair in the $L \times L$ square network is $\sqrt{2} \Psi / 3$, the average hop count is $\sqrt{2} \not / 3 d$. Therefore, $T_{e \psi}$ is

$$
T_{e \psi}=\frac{2 L^{2} b \psi}{\sqrt{3} \not_{\min }^{2}} \div \frac{\sqrt{2} \not \psi \psi}{3 d}=\frac{\sqrt{6} \not b d \psi}{D_{\text {n }}^{2}(\text { in }} \text { when } d_{C S \psi}<D_{\text {min }}
$$

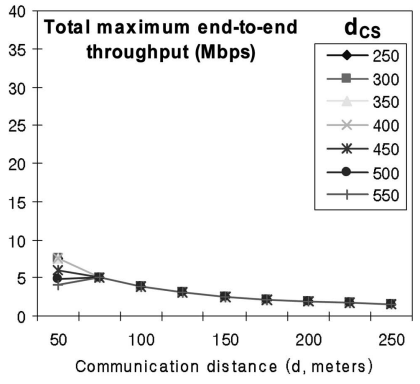

(a)

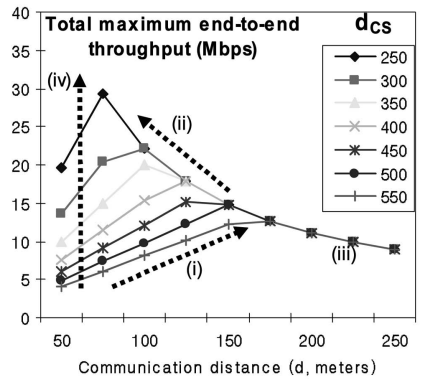

(b)
Fig. 4. Maximum total end-to-end throughput ( $b=1 \mathrm{Mbps}, L=10 \mathrm{~km}$, $z_{0}=10 \mathrm{~dB}$ ). (a) $\alpha \notin 2.0$. (b) $\alpha \notin 4.0$.

provided the wireless communication bandwidth is blbits/ second and $d_{C S \psi}$ corresponds to the radius of the CS zone. Equation (5) becomes clearer if we make a simplifying assumption that the six interferers are all $D$ lapart from the receiver $j$. Then, (3) and (5) become

$$
\frac{d^{-\alpha \psi}}{6 D_{\min }^{-\alpha \psi}}=z_{0} \Rightarrow D_{\min }=\leftarrow \sqrt[\alpha]{6 z_{0}} \phi \psi
$$

and

$$
T_{e \psi}=\frac{\sqrt{6} \not b d \psi}{\left(\sqrt{6} x_{\rho} \phi\right)^{2}}=\stackrel{\sqrt{6} \not b \psi}{\left(\sqrt[a]{6} z_{p}\right)^{2}} \times \frac{1}{d} \text { when } d_{C S \psi}<D_{\min } \cdot \psi \quad(7) \leftarrow
$$

In other words, $T_{e \psi}$ increases as the communication distance $d \psi$ decreases as predicted in [4]. For example, in the $915 \mathrm{MHz}$ WaveLAN radio hardware, a $d_{C S \psi}$ of $550 \mathrm{~m}$ is considered optimal using (6) when the communication distance is about $198 \mathrm{~m}\left(=550 \mathrm{~m} /\left(6 z_{0}\right)^{1 / \alpha 2 /}\right.$ when $\alpha \psi 4.0$ and $z_{0}=10 \mathrm{~dB}$ ). Note that, when $d_{C S \psi} \geq D_{\min }$, senders would be separated by $d_{C S}$ instead of $D_{\min }$ due to carrier sensing and, thus, (5) becomes

$$
T_{e \psi}=\frac{\sqrt{6} \Psi b d \psi}{d_{d S \psi}^{2}}=\frac{\sqrt{6} \Psi b \psi}{d_{C}^{2} \psi \psi} \times d \text { when } d_{C S \psi} \geq D_{\min }, \psi
$$

meaning that $T_{e \psi}$ increases as the communication distance $d \psi$ increases.

Fig. 4 shows $T_{e \psi}$ versus $d$ for different $d_{C S \psi}$ values based on (3), (5), and (8). When the path loss exponent is 2, the effect is not significant, as in Fig. 4a. However, when it is 4 , as in a land mobile environment, the effect becomes significant as in Fig. $4 \mathrm{~b}$. From the $d_{C S}$ 's point of view, when $d_{C S}$ is large enough, it is better to exploit the CS-protected area and deliver data packets as far as possible within the CS zone (large communication distance $d$ ). See mark (i) in Fig. 4b. When $d_{C S \psi}$ is not large, we can obtain a better performance by shortening the communication distance even though it increases the hop count between the source and the destination (mark (ii)). From the communication distance's perspective, when short communications are frequent, the $D_{\min }$ required is smaller and (8) applies. $T_{e}$ increases as $d_{C S \psi}$ decreases (or less sensitive carrier sensing) as indicated by (iv) in the figure.

The next section proposes a new MAC algorithm, which $(5) \leftarrow$ uses a smaller $d_{C S \psi}$ and adjusts the communication distance whenever necessary. We adopt a fixed $d_{C S \psi}(350 \mathrm{~m})$ in the 


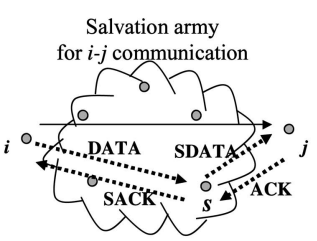

(a)

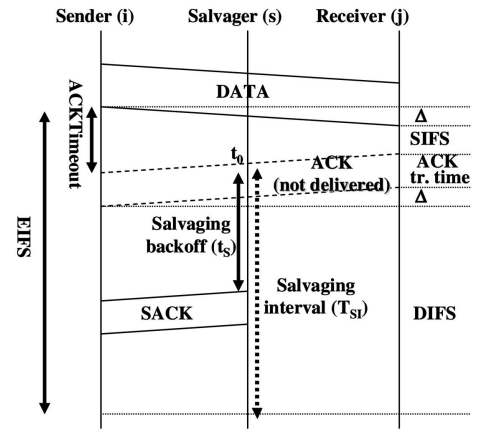

(b)

Fig. 5. Salvation army and salvaging procedure. (a) Salvation army for $i-j$ communication. (b) Distributed selection of a salvager.

proposed scheme because the adaptive adjustment of this value would be too complex to be useful [27].

\section{MASA: HOP-BY-HOP MAC-LAYER SALVAGING}

One important observation from the previous section is that the performance of a multihop network greatly depends on the CS threshold and communication distance. This section proposes the Multiple Access with Salvation Army (MASA) protocol that uses a fixed, higher CS threshold (smaller $d_{C S}$ ) to increase the spatial reusability and solves the collision problem from hidden terminals via packet salvaging. It essentially reduces the communication distance on the fly by breaking one-hop communication into two smaller-hop communications when it is beneficial. It is based on the DCF but does not use the optional RTS/CTS exchange because collisions in the absence of RTS/CTS can also be effectively masked by packet salvaging. The MASA algorithm includes two new frame types, called SACK (Salvaging $A C K$ ) and SDATA (Salvaging DATA) as will be explained later in this section. Throughout this paper, we assume PHY (physical layer) and MAC parameters of $915 \mathrm{MHz}$ WaveLAN radio hardware [18], which are also used to derive default parameters in the ns-2 network simulator [17].

\subsection{Packet Salvaging in MASA}

In wireless networks, nodes use broadcast as opposed to point-to-point communication and, therefore, data packets are typically sent to multiple nodes in the proximity of the sender at no extra cost. We call the set of those overhearing nodes the salvation army. A key idea in the proposed MASA protocol is that a third party node (say, node $s$ ) in the salvation army captures or salvages a data packet that collided at the intended receiver and lets the packet make progress toward the receiver. This is shown in Fig. 5a. Since the sender-salvager distance is smaller than the senderreceiver distance, the salvager $s \psi$ receives the packet successfully with a higher probability and completes the communication session by replying SACK to node $i$. It then forwards the data packet (SDATA) to the original receiver $j \psi$ based on the usual defer and backoff procedure. Note that, while ACK is transmitted regardless of the status of the medium, SACK is transmitted only when the medium is free. This is to address the potential collision problem. The

modified MAC behaviors at the salvager $(s)$, the sender $(i)$, and the receiver $(j)$ tare described below.

First, at the sender $(i)$, when an ACK is not received during the ACKTimeout interval, the sender concludes that the transmission has failed and invokes its backoff procedure to retransmit the packet in DCF. In MASA, the sender cancels the backoff procedure when it receives SACK even after the ACKTimeout interval and does not retransmit the packet. Second, at the salvager $(s)$, it waits for an SIFS (short interframe spacing) [21] upon successful reception of a data packet and checks the channel status (BUSY or IDLE) using the clear channel assessment or CCA signal supported by the IEEE 802.11-conformant hardware [21]. This is to determine whether it is necessary to salvage the packet or not. If ACK is received (more accurately, if the channel status changes to BUSY), it cancels its salvaging activity. Otherwise, it starts its salvaging backoff procedure (to be explained shortly) and accordingly transmits SACK to the sender. Then, it starts its normal backoff procedure to forward the data packet (SDATA) to the receiver $(j)$, who then replies with an ACK to the salvager after an SIFS period. Both the sender and the salvager will retransmit the same packet a prespecified number of times as defined in the DCF if they do not receive ACK or SACK. Note that MASA does not allow a salvaged packet to be salvaged again, which is an improvement over the earlier version of MASA [1]. This is because consecutive salvages of a packet make it travel along a longer, detour path, potentially losing the benefit of MASA. Third, at the receiver $(j)$, it may receive the same data packet more than once from multiple salvagers. We explain below how this problem is handled in MASA.

When more than one node salvages the same packet, the receiver receives duplicate packets. They can be filtered out within the receiver MAC based on the original functionality of the DCF, called duplicate packet filtering [21]. This algorithm matches the sender address (Addr2 in Fig. 6) and the sender-generated sequence control number (SC) of a new packet against those of previously received ones. If there is a match, the receiver transmits ACK but does not forward the packets. This does not solve the abovementioned problem in MASA because duplicate packets from different salvagers ( $s$ and $t$ ) include different identities from node $i$ in the Addr2 field. Our approach in MASA is to use a new data type SDATA that includes the original sender's address in Addr4 (logical address field) so that the receiver can use this address rather than the salvager address (Addr2) when it compares against the stored information.

\subsection{Determination of a Salvager among the Salvation Army}

When more than one node is able to salvage a collided packet, the candidate that can make the greatest progress should be selected. For this purpose, we assume that each node maintains a neighbor list and signal quality information for its neighbors. It is not difficult to keep track of its neighbors because each node overhears every other neighbor's communications. The signal quality for each neighbor can be obtained using the previous signal it received from the particular neighbor. We modify the functionality of PHY layer of IEEE 802.11 to support this. The PHY layer of IEEE 802.11 checks the Received Signal 


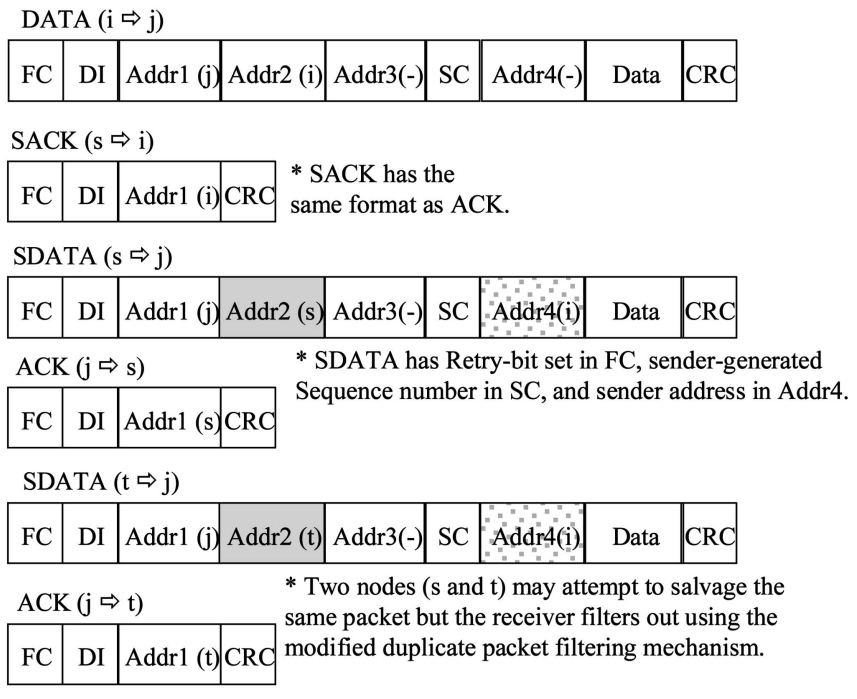

Fig. 6. Format of MPDU frames in the MASA protocol (MPDU: MAC protocol data unit, FC: Frame control, DI: Duration/Connection ID, SC: Sequence control).

Strength (RSS) of the carrier to inform the channel status to the MAC layer (CCA signal) [21]. In MASA, the PHY layer is assumed to inform not only the channel status, but also the RSS information to the MAC. When a sender transmits a MAC frame, we assume that the frame includes the signal quality information for the receiver.

Now, when node $s \psi$ receives a data packet that is not intended for it, the node evaluates its eligibility as a salvager using the following rules:

1. The specified sender, as well as the receiver, must be in the neighbor list of node $s$.

2. When node sloverhears a SACK for the packet it is about to salvage, it should cancel its salvaging activity for that particular packet.

3. In order for node $s \psi$ to make progress toward the receiver, it must be nearer the receiver than the sender. Node $s /$ speculates this condition based on signal strength information as mentioned earlier.

4. Node sumust not have a pending packet at its MAClayer software.

5. Node $s$ loes not have a recent history that it failed to forward a packet after salvaging for the same pair of nodes. The neighbor list mentioned earlier can be used for this purpose as well. This is important because a node, ignorant of a broken link, might keep on salvaging packets but fail to forward them. This was not clearly stated in our earlier work [1].

If a node is considered a legitimate candidate, it starts its salvaging activity at time $t_{0}$ after waiting for an ACKTimeout interval as shown in Fig. 5b. Then, it chooses its salvaging backoff time $\left(t_{S}\right)$ within the salvaging interval $\left(T_{S I}\right)$ during which it is allowed to salvage the packet.

- $T_{S I y}$ can be considered the opportunity window open to salvagers, which starts at $t_{0}$ and must end before the next data transfer begins. Based on the operation principle of the $\mathrm{DCF}$,

$$
T_{S I \psi}=A C K \psi \text { transmission-time }-+D I F S, \psi
$$

as shown in Fig. 5b. This is because nodes in the proximity of the communication between nodes $i \psi$ and $j$ would wait for ACKTimeout for allowing the pair to complete their communication. An additional DIFS is available because it is required for a new data transfer to start. Nodes outside of $T R_{i \psi}$ may corrupt the SACK packet by transmitting their own during salvaging. However, based on the DCF specification, they would wait EIFS (Extended IFS) before starting their own transmission [21], which turns out to offer the same opportunity window to salvagers because EIFS is set to

\section{SIFS\& ACKutransmission-time- + DIFS $\psi$}

[21], [16]. For simplicity, we do not include the propagation delay, $\Delta$, which is relatively small and can be ignored.

- $\quad t_{S \psi}$ is considered a priority among multiple candidates. The node that is closer to the receiver should be elected as the salvager because it can make greater progress. The proposed MASA uses the signal quality to determine the salvager. In other words, node $s$ ccalculates $t_{S}$, at which it transmits a SACK using the signal quality from the sender $\left(q_{i s}\right)$ and that from the receiver $\left(q_{j s}\right)$, i.e., $t_{S \psi}=q_{i s} / q_{j i \psi} \times T_{S I}$. This is based on the assumption that the signal quality directly corresponds to distance. Even if the assumption is not valid, this arbitration rule still works well and it simply becomes a randomized algorithm.

Fig. 7 summarizes the proposed MASA algorithm.

\section{Simulation and Evaluation}

The performance of the MASA algorithm is evaluated using the ns-2 [16], which simulates node mobility, radio network interfaces, and the DCF protocol. The two-ray ground propagation channel is assumed with a radio transmission range of $250 \mathrm{~m}$ and a data rate of $2 \mathrm{Mbps}$. In order to show the benefit of the packet salvaging, Section 5.1 presents the simulation result of a simple 4- and 5-node scenario with a single interferer. More realistic scenarios with more than 50 nodes and the corresponding simulation results are presented in Sections 5.2 and 5.3, respectively.

\subsection{Benefit of Packet Salvaging with a Single Interferer}

Fig. 8 shows a simple communication scenario with four and five nodes. A pair of nodes $i$ land $j$ lare our primary focus while another node pair $A-B$ iprovides interfering signals. Node $i$ ssends 512 -byte constant bit rate (CBR) or TCP packets to node $j$. Node Azalso sends 512-byte CBR or TCP packets to node $B$. In the direct scenario in Fig. 8a, there exists no salvager candidate between nodes $i \psi$ and $j \psi$ and, thus, SIR at node $j \psi$ is always low and the communication is easily subjective to interference from node $A$. On the other hand, in the salvaging scenario in Fig. 8b, node $s \psi$ is capable of capturing and salvaging a packet collided at node $j$. Thus, node $j$ receives a stronger signal with high SIR from node $s$. Using (2), SIR at node $j \psi$ in the direct 


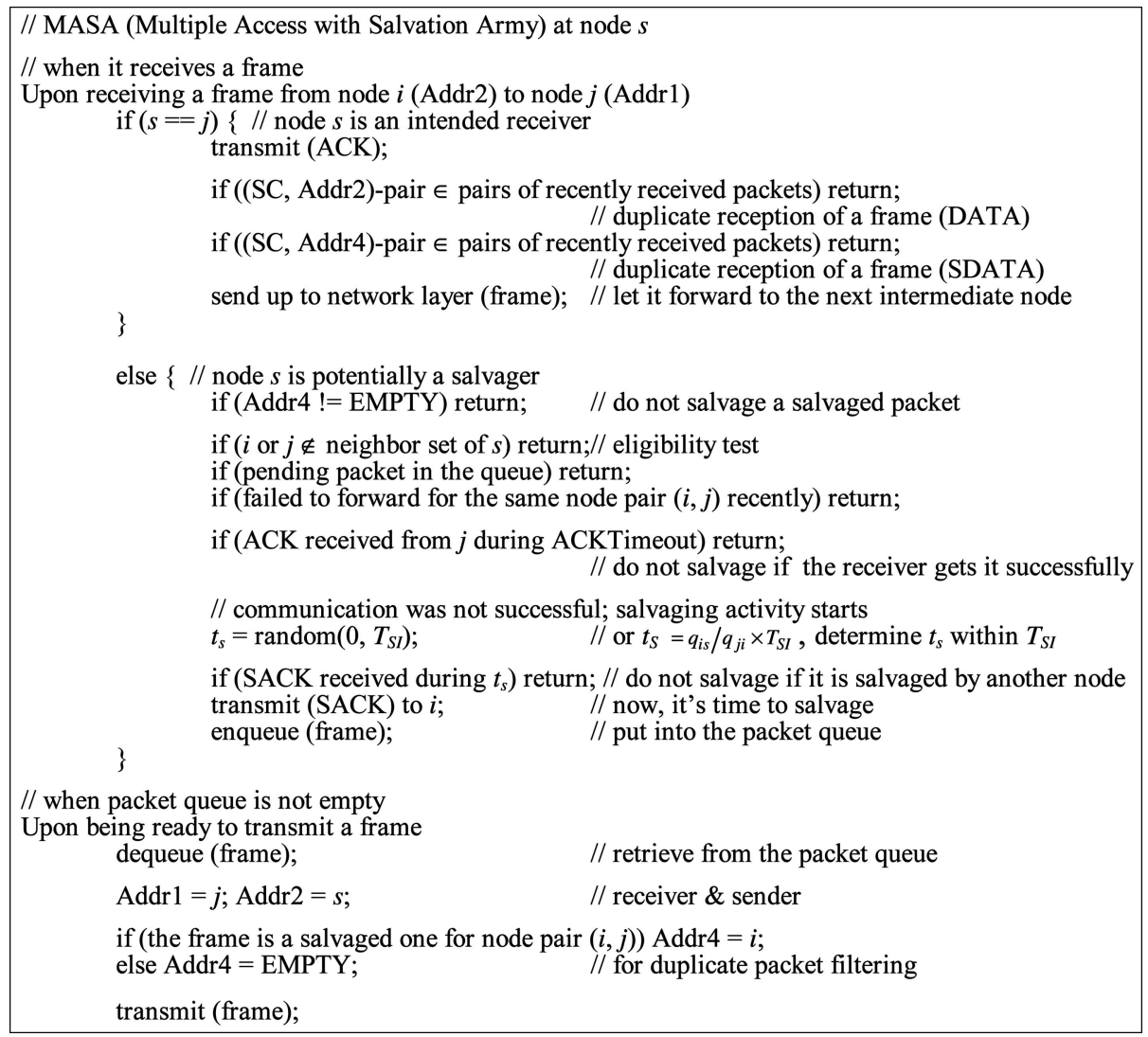

Fig. 7. The MASA algorithm.

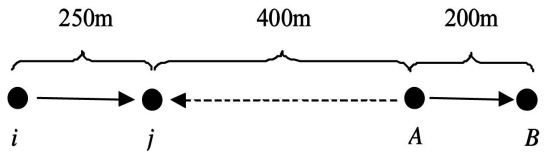

(a)

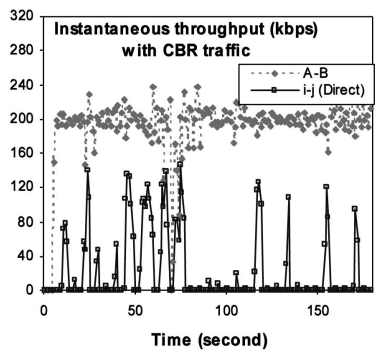

(c)

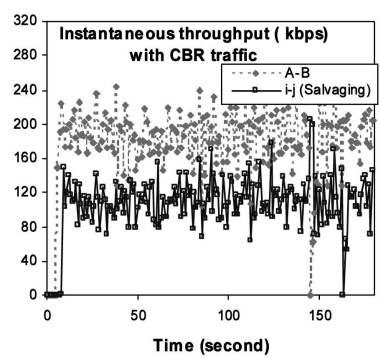

(d)

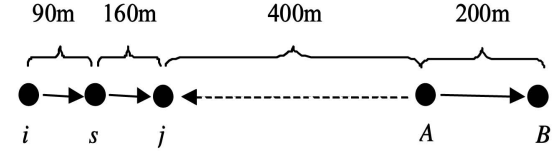

(b)

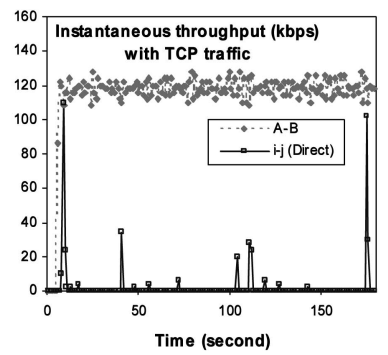

(e)

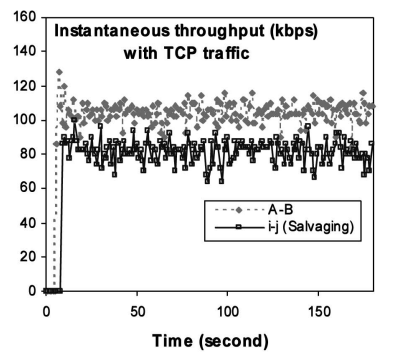

(f)

Fig. 8. The effect of packet salvaging with simple communication scenarios. (a) Direct scenario. (b) Salvaging scenario. (c) Direct with CBR. (d) Salvaging with CBR. (e) Direct with TCP. (f) Salvaging with TCP.

scenario is $(400 / 250)^{4}$ or $8.16 \mathrm{~dB}$ for the packet from node $i$, which is smaller than $z_{0}$. But, in the salvaging scenario, it is $(400 / 160)^{4}$ or $15.92 \mathrm{~dB}$ for the packet that has been salvaged by $s$, which is larger than $z_{0}$.

Figs. 8c, 8d, 8e, and $8 \mathrm{f}$ compare instantaneous throughput, measured at every simulated second, with CBR and TCP traffic. As shown in Figs. 8c and 8d, the salvaging scenario offers a higher aggregate throughput than the direct scenario with CBR traffic even though the average number of hops between the communication pair $(i-j)$ is larger. This is also true with TCP traffic as drawn in Figs. 8e and 8f. Moreover, the direct scenario exhibits unacceptably serious unfairness, which is a well-researched phenomenon observed by $\mathrm{Xu}$ and Saadawi [30]. According to their observation, the throughput of one TCP session can be almost zero while the other TCP session monopolizes the channel bandwidth. Our simulation results confirm that this is also the case with CBR traffic and infer that the capture effect and packet salvaging may alleviate the fairness as well as the performance problem. 


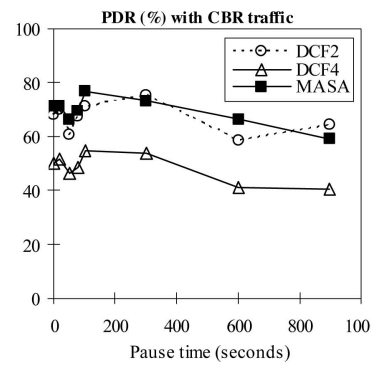

(a)

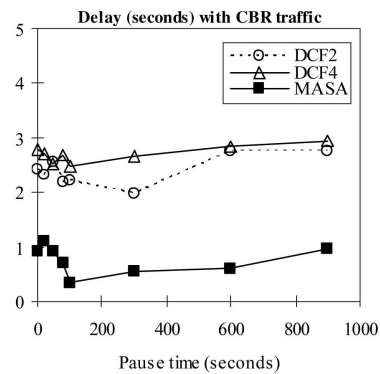

(b)

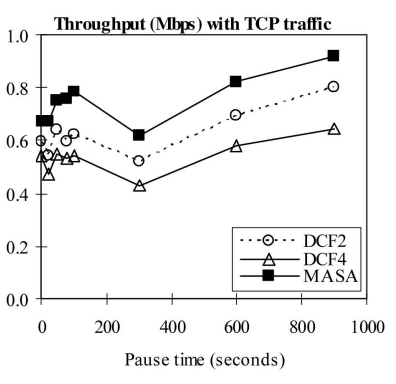

(c)

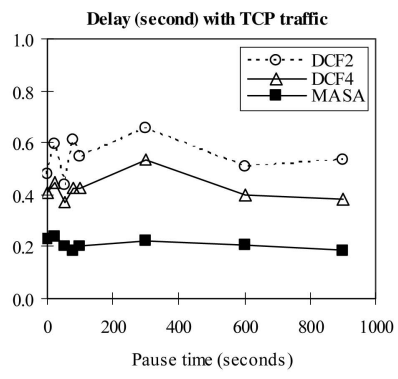

(d)

Fig. 9. Performance comparison with mobility. (a) PDR with CBR. (b) Packet delay with CBR. (c) Throughput with TCP. (d) Packet delay with TCP.

\subsection{Simulation Environment with Multiple Interferers}

The previous subsection shows the benefit of packet salvaging in MASA on a small network with a single interferer. The following two subsections present the merits of the proposed MASA algorithm in more complex and larger network scenarios. Protocols to be compared are MASA, DCF2 (DCF without RTS/CTS), and DCF4 (DCF with RTS/CTS). We included DCF2 because MASA does not incorporate the RTS/CTS handshake either. Note that DCF2 in general outperforms DCF4, which is counterintuitive but has been predicted by a number of researchers [28], [29] and also has been discussed in Section 3.1 in this paper. We observed, however, that DCF2 degrades more significantly in comparison to DCF4 with the shadowing radio-propagation model. Randomness in radio propagation makes the RTS/CTS handshake more useful. We will discuss this issue later in Section 5.3.

Our evaluation is based on the simulation of 100 mobile nodes located in an area of $300 \times 1500 \mathrm{~m}^{2}$. The CS distance is assumed to be $550 \mathrm{~m}$ and $350 \mathrm{~m}$ with the DCF and the MASA, respectively. The AODV routing algorithm [7] is used to find and maintain the routes between two endnodes. The data traffic simulated is CBR and TCP traffic. In case of CBR, 40 sources generate three 256-byte data packets every second. Destination nodes are selected randomly. The random waypoint mobility model is used in our experiments with the maximum node speed of $5 \mathrm{~m} / \mathrm{s}$ and the pause time of $0 \sim 900$ seconds. The simulation is run for 900 seconds and each simulation scenario is repeated 10 times to obtain steady-state performance metrics. For more accurate performance evaluation, we also used different routing algorithms (DSR [6]) and different propagation models. Various traffic intensities in terms of packet rate and the number of sources and various numbers of nodes are also used to observe the performance scalability of the DCF and the MASA.

In our experiments, we assume the following aspects of signal capture:

- When two packets arrive, if the first signal is $10 \mathrm{~dB}$ $\left(z_{0}\right)$ stronger than the second, then the first signal can be successfully received. However, if the second signal is $10 \mathrm{~dB}$ stronger than the first, neither packet is successful because the receiving node already started decoding the first signal and cannot switch to the second immediately. This is actually the way the ns-2 is implemented. However, in the latter case, if the first signal is weaker than the receive threshold but larger than the CS threshold, the receiver can receive the second signal successfully. Since ns-2 still drops both packets in this case, we modified ns-2 to reflect this fact.

- The SIR computation requires two samples of the signal, the desired signal and the signal with interference, and their availability is assumed for computation.

- The signal strength comparison for determining capturing is on a per-packet basis in ns-2. That is, if multiple interfering packets were to be received, they are only compared individually, not in their combinations. We modified ns-2 to simulate additive interference if there exist concurrent multiple interfering signals.

\subsection{Results and Discussion}

Fig. 9 shows the network performance with respect to node mobility represented by pause time. Note that 900 seconds of pause time means a static scenario while 0 seconds means a constant-moving scenario. Figs. 9a and 9b show the packet delivery ratio (PDR) and packet delay with CBR traffic. While the PDR of MASA is on par with that of DCF2, as shown in Fig. 9a, it is clear from Fig. 9b that MASA outperforms DCF2 and DCF4 in terms of packet delay, showing $53 \sim 85$ percent and $59 \sim \$ 6$ percent reduction, respectively. A major factor that contributes to reduction in packet delay is fewer false alarms for live links. Each link error report in AODV triggers a route-discovery procedure, causing the packets in transit as well as the following packets to experience a large delay until a new routing path is found. It also causes network-wide flooding of RREQ packets that waste a substantial amount of wireless bandwidth.

The large reduction in packet delay with the CBR traffic motivated us to experiment with TCP traffic because TCP behaves adaptively according to a round trip time (RTT) estimate. We simulated 40 TCP connections in the same ad hoc network environment. The aggregate end-to-end throughput and response time are plotted in Figs. 9c and $9 \mathrm{~d}$, respectively. As shown in the figures, the MASA achieves as much as 27 percent and 45 percent higher throughput than DCF2 and DCF4. The response time is reduced by 70 percent and 58 percent, respectively, as seen 


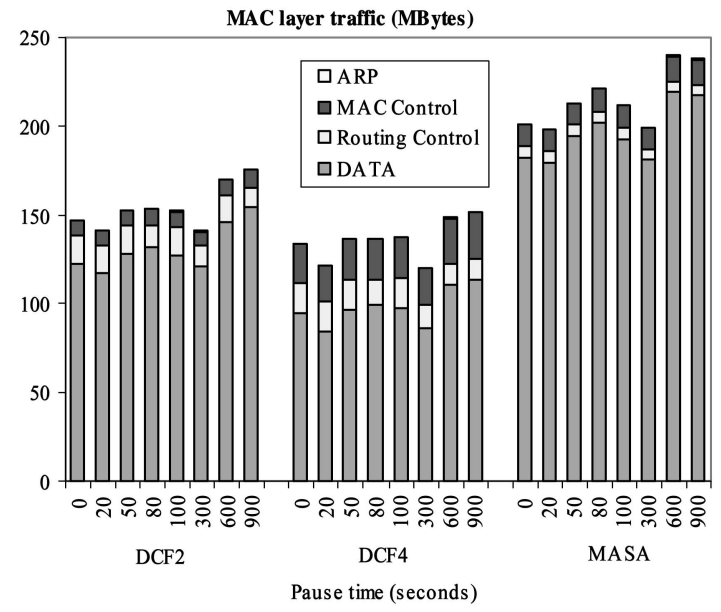

(a)

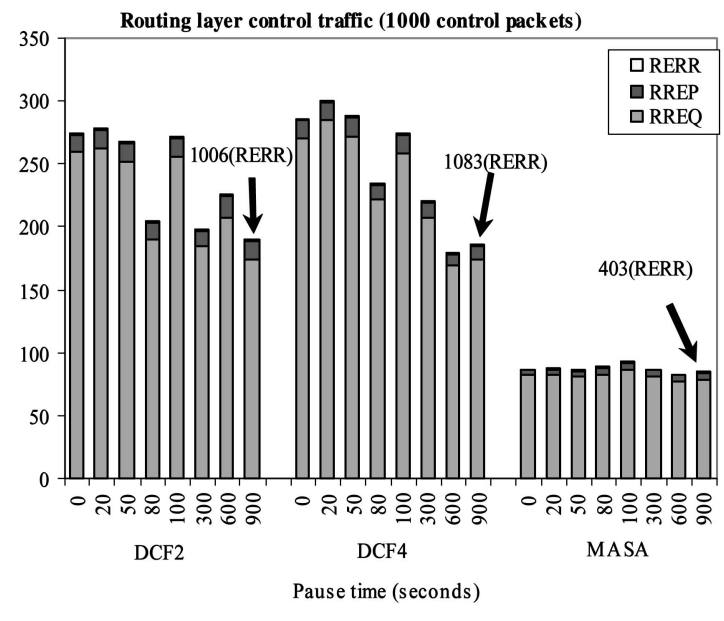

(b)

Fig. 10. Overhead analysis with TCP traffic. (a) MAC layer overhead. (b) Routing layer overhead.

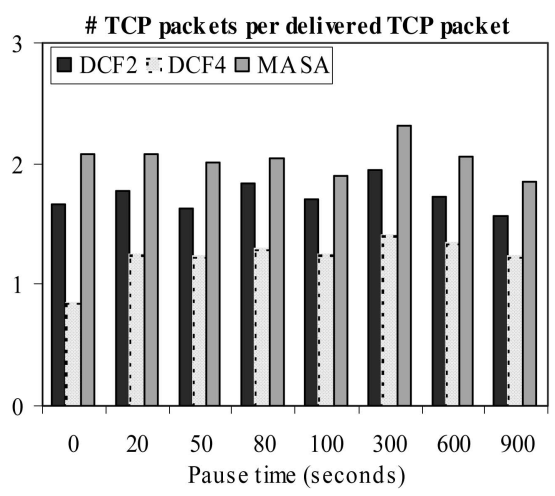

(a)

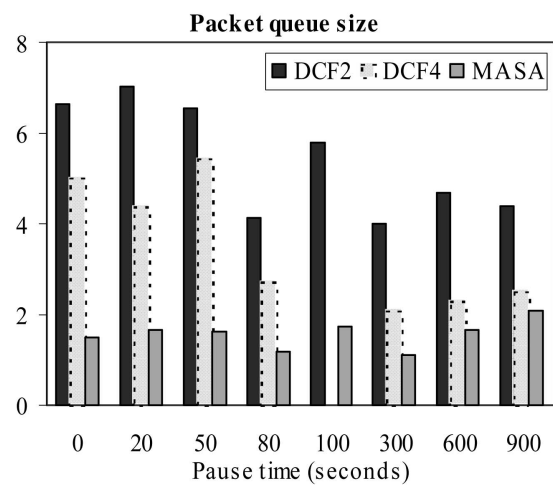

(b)

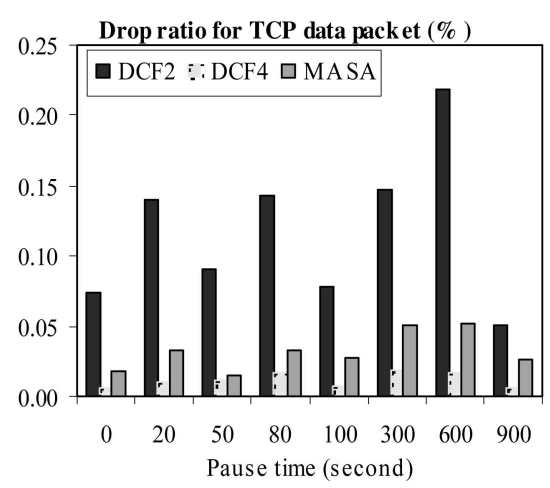

(c)

Fig. 11. Another overhead analysis with TCP traffic. (a) Normalized data overhead. (b) Packet queue size. (c) Drop ratio.

in Fig. 9d. It is concluded from Fig. 9 that the MASA protocol and its MAC-layer packet salvaging mechanism in general improve the network performance, particularly for TCP-based applications. More importantly, the MASA would be best suited in application scenarios where delay is a primary concern.

MAC and routing overhead, data overhead, and packet queue size have been measured during the simulation. Fig. 10 shows that the overhead analysis results with TCP traffic. First, Fig. 10a presents various overhead traffic: ARP (address resolution protocol) traffic (almost negligible), MAC layer control traffic (RTS, CTS, and ACK), routing control traffic (RREQ, RREP, and RERR) and DATA traffic (TCP data and TCP Ack). Since MASA encourages more concurrent transmissions due to its lower carrier sense range, it shows more DATA traffic, indicating that MASA uses more bandwidth for useful data transmission than DCF2 and DCF4. For instance, with a pause time of 0 seconds, data traffic takes up 91 percent of the entire traffic in case of MASA, while it is 83 percent and 71 percent in DCF2 and DCF4, respectively. Like DCF2, it shows less MAC layer control traffic than DCF4 because it does not use the RTS/CTS handshake. MASA generates the least routing control traffic, which is detailed in Fig. 10b.
In Fig. 10b, DCF2 and DCF4 generate more than 3.4 and 4.1 times more routing control traffic than MASA, respectively. (It is 1.3 and 7.1 with CBR traffic.) At the pause time of 900 seconds, where mobile nodes are static and, thus, no RERR packets are expected, DCF2 and DCF4 still result in 1,006 and 1,083 RERR packets, which must be contrasted to 403 such packets with MASA. Making progress with packet salvaging in the MASA algorithm reduces false alarms by more than half in spite of network congestion and, thus, reduces the routing control overhead significantly. On average, MASA employs $0.27 \quad 0.36$ control packet per successfully delivered data packet, while it is $0.59 \quad 1.22$ and 0.721 .48 with DCF2 and DCF4, respectively.

However, as far as the data transmission overhead (retransmissions) is concerned, MASA is disadvantageous. Fig. 11a shows the number of TCP packets transmitted at the MAC layer for each successfully delivered TCP packet. They are 1.65, 0.84, and 2.08 packets for DCF2, DCF4, and the MASA, respectively, with a pause time of 0 seconds. Since the DCF4 algorithm employs the RTS/CTS exchange before transmitting a data packet, it results in fewer collisions on data packets and, thus, reduces the number of retransmissions compared to DCF2 and MASA. In comparison with DCF2, the MASA algorithm incurs more 


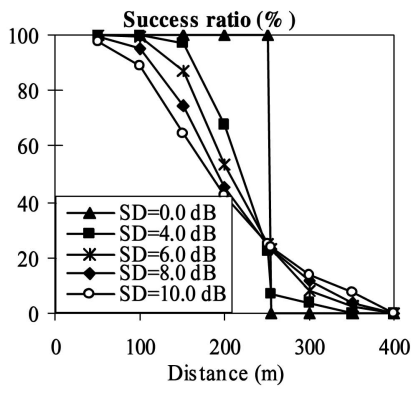

(a)

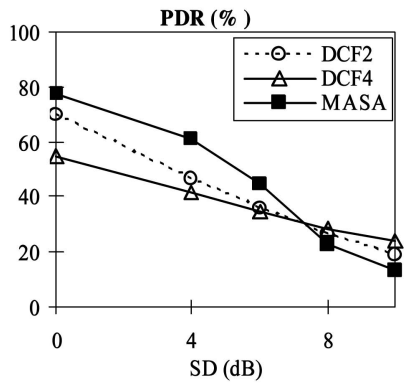

(b)

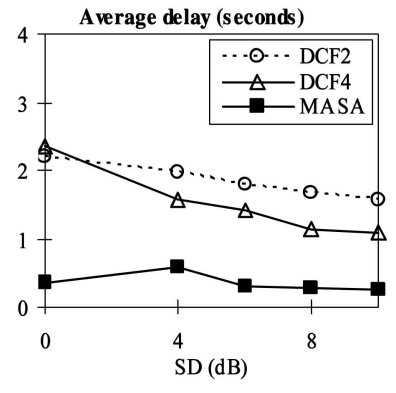

(c)

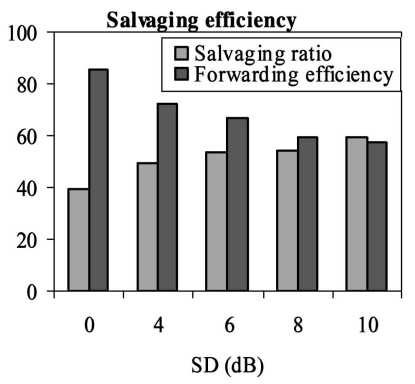

(d)

Fig. 12. Performance with shadowing model with CBR traffic. (a) Success ratio with distance. (b) PDR versus SD. (c) Delay versus SD. (d) Salvaging efficiency.

overhead mainly because of the reduced CS zone. Nonetheless, it does not overshadow the advantage of MASA as already seen in Fig. 9.

A primary advantage of MASA is short packet delay. Our investigation shows that the packet queuing delay is an important ingredient for this. Once again, making progress via packet salvaging facilitates a mobile node to quickly offload pending packets and, therefore, it helps keep its packet queue at the routing layer as short as possible. In each of 900 seconds of simulation runs, we collected the information of packet queue size every 10 seconds at each node and calculated the average statistics across all mobile nodes in the network. As shown in Fig. 11b, each node has about 5.39 and 3.06 packets in its queue on average with DCF2 and DCF4, respectively, while it is 1.57 with MASA. Similar observations have been made with CBR traffic.

Fig. 11c shows the number of TCP data packet drops at the MAC layer in terms of the ratio of packet drops over the total number of attempts. A TCP data packet is dropped when all of the predetermined number of retransmissions (e.g., four) fail. DCF4 outperforms the other protocols because it uses the RTS/CTS exchange before sending a TCP data packets. (However, DCF4 drops a large number of RTS packets instead.) MASA performs on par with DCF4 and much better than DCF2. Therefore, it can be concluded that MASA achieves the same level of drop ratio without incurring the overhead caused by the RTS/CTS exchange.

Recent experimental studies show that the shortest (hop count) path does not always provide the best performance because it usually consists of longer hop communications, each of which is easily subjective to interference with a small SIR [13], [14], [15]. In order to see how MASA performs in a more realistic environment, a set of experiments has been conducted with the shadowing propagation model instead of the conventional two-ray ground propagation model introduced in Section 2.1. Shadowing is caused by the lack of visibility between two communicating nodes and it causes slow variations over the mean received power. The mean received power is calculated deterministically based on the communication distance. The randomness of the channel is described by a log-normal random variable, the distribution function of which is Gaussian with zero mean and a specified standard deviation (SD). MASA is expected to be more advantageous over a random channel because of its adaptivity.
Before presenting the simulation results, Fig. 12a shows how the radio channel behaves with the shadowing model presenting the success ratio versus communication distance using ns-2. In case of SD of $0.0 \mathrm{~dB}$, the shadowing model is equivalent to the deterministic two-ray ground model and, thus, the success ratio is 100 percent if the distance is less than $250 \mathrm{~m}$, which is the transmission range. Otherwise, it is 0 percent. As SD increases, more communications fail even if the distance is less than $250 \mathrm{~m}$, and more communications succeed even if the distance is longer than $250 \mathrm{~m}$. When the communication distance is $200 \mathrm{~m}$, the success ratio is 42 percent with SD of $10 \mathrm{~dB}$. Less than a half of the transmission attempts can be successful even if the communication distance is shorter than the transmission range.

Figs. $12 \mathrm{~b}$ and $12 \mathrm{c}$ show the effect of channel randomness on the network performance such as PDR and packet delay with the CBR traffic. MASA consistently outperforms DCF2 and DCF4 in terms of packet delay as shown in Fig. 12c. However, this is not always the case with PDR as shown in Fig. 12b. It loses its advantage when SD becomes extremely large, such as $10 \mathrm{~dB}$.

This can be explained with Fig. 12d. Since MASA salvages collided packets, it would be interesting to know how many packets are actually salvaged and how many of them are successfully forwarded to the original receivers, which we call salvaging ratio and forwarding efficiency, respectively. Fig. 12d shows that the salvaging ratio and forwarding efficiency are about 40 percent and 80 percent, respectively, when SD is $0 \mathrm{~dB}$. More than a third of the packets are salvaged (since they are collided) and most of them are forwarded successfully, demonstrating the effectiveness of the MASA algorithm. When SD is $10 \mathrm{~dB}$, the salvaging ratio is as high as 59 percent but the forwarding efficiency is as low as 57 percent. Only a half of the salvaged packets are forwarded successfully due to the low success ratio, e.g., 42 percent, as explained earlier. Even though some of the lost packets are salvaged and forwarded successfully to the next-hop node $(59 \% \times 57 \%=33.6 \%$ of packets), many others are ultimately lost in spite of neighbors' help to salvage them. Their help in this case makes the channel contention even worse, decreasing the network performance without yielding any benefit. Packet salvaging does not help when $\mathrm{SD}$ is $10 \mathrm{~dB}$ but the performance benefit of MASA is observed up to a SD of $8 \mathrm{~dB}$. The packet delay in Fig. 12c decreases when the 
network environment is more random. This should not be interpreted as an improvement because fewer packets are delivered to the desired destinations.

\section{Related Work}

The MASA algorithm essentially favors the use of higherquality links, which, in most cases, are short-distance. An advantage of short communications in a multihop environment has been reported in the literature. Grossglauser and Tse concluded in [4] that the network capacity can be maximized by allocating the channel to the nodes that can communicate over a short distance. In their proposed algorithm, each sender buffers the data traffic until its destination node approaches near it. Similarly, De Couto et al. observed that the shortest (hop count) path does not always provide the best performance because this path usually consists of longer-hop communications, each of which is easily subjective to interference with low radio link quality or a small SIR [14]. This section overviews existing packet-salvaging schemes at the network and MAC layer in Sections 6.1 and 6.2, respectively, and other throughputenhancing techniques in Section 6.3.

\subsection{Packet Salvaging at the Network Layer}

For a collided packet, a possible solution at the network layer is to relay it via an alternative path to avoid the congested area and to exploit unused area. This may improve the performance significantly because a link breakage, even though it is temporary, could cause serious performance degradation if it is misinterpreted as a permanent link error. A number of packets already in flight could be lost and a routing protocol, e.g., DSR [6], would initiate a new route-discovery procedure that basically floods the network with control messages, making the situation worse or the problem persist longer.

In DSR, an optimization technique known as "packet salvaging" [6] is used so that the node encountering the forwarding failure may search its local storage for alternative routes. If a route is found, it is used to forward the undeliverable packets without resorting to an expensive route-discovery procedure. The "local repair" mechanism in the AODV routing protocol [7] does a similar thing. Valera et al. suggested a distributed packet salvaging scheme for more improvement [31]: Every node maintains a small buffer for caching data packets that pass through it and at least two routes to every active destination. When a downstream node encounters a forwarding error, an upstream node with an alternative route as well as the pertinent data in its buffer can be used to retransmit the data packets.

However, the above-mentioned packet-salvaging schemes do not keep the sender from initiating an expensive route-discovery procedure because their original goal is to save packets in flight. Moreover, these schemes either kick in only after a lower-level protocol has attempted a number of times without a success. For example, the DCF [3] retransmits four times before the link error is reported to the higher-level protocol. Each retransmission not only wastes resources such as node energy and channel resource, but also extends the packet delay. Shortest-path routing protocols aggravate the situation because they prefer a longer per-hop communication distance, and the corresponding wireless links are more prone to temporary breakages [14].

\subsection{Packet Salvaging at the MAC Layer}

Nondeterministic packet salvaging at the MAC layer has recently received significant attention to deal with frequent, temporary link errors quickly and efficiently [8], [9], [10], [11], [12]. This is a more direct and efficient approach because each hop connection is established for communication at the link layer. This subsection overviews four MAClayer packet salvaging schemes:

- $\quad$ Biswas and Morris proposed Extremely Opportunistic Routing (ExOR), which defers the choice of the nexthop node among the precomputed candidates until after the previous node has transmitted the packet via its radio interface [8]. Based on the number of hops to the final destination and the past history of delivery ratios, the sender prioritizes the candidates and includes the list in the packet header. Each candidate competes to become a receiver by delaying its reply for the amount of time determined by its priority in the list.

- Blum et al. proposed Implicit Geographic Forwarding (IGF), which is also a nondeterministic algorithm [9]. Like in Geographic Forwarding (GF) [32], the sender has position information of its neighbors as well as the final destination node of its packet. However, unlike in GF, the choice of the next-hop node is not determined by the sender but by competition among the candidates as in the ExOR scheme. The sender transmits an Open RTS (no intended receiver is specified) and each candidate delays its response (Clear-to-Send or CTS) for an amount of time determined by the distance to the destination and the remaining node energy.

- Zorzi and Rao presented Geographic Random Forwarding (GeRaF), which is basically the same as IGF, but the competition is coordinated by the sender with two control messages, called CONTINUE and COLLISION, in addition to RTS and CTS messages [12]. Here, the transmission coverage area of a sender, only in the direction of the final destination, is divided into a number of regions. When a sender transmits an RTS, any node in the closest region to the destination responds with a CTS. When no CTS is heard, the sender transmits a CONTINUE message so that the nodes in the next region can respond. When more than one CTS are sent, the sender hears a signal but is unable to detect a meaningful message. In this case, the sender transmits a COLLISION message, which will trigger a collision-resolution algorithm [12].

- In the Stateless Nondeterministic Geographic Forwarding (SNGF) algorithm, which is part of the sensor network protocol SPEED [10], each node computes the forwarding candidate set for each destination, a member node of which is a neighbor and is closer to the destination than the node itself. Location 
information of the node as well as the destination is necessary in SNGF.

However, the above-mentioned schemes depend either on location information [9], [10], [12] or use a link-state flooding scheme [8] to help determine the salvager among multiple candidates, which may not be feasible in real implementations. The MASA algorithm presented in this paper is a practical nondeterministic MAC algorithm that requires neither the location information nor the link state propagation. Note that MAC-layer packet salvaging targets temporary link breakages, assuming that the current routing path is still usable, while network-layer packet salvaging attempts to save packets in transit (and initiates a new route discovery as in a conventional routing algorithm), assuming that the routing path is no longer usable. If a communication attempt fails due to a short-lived temporary problem, a new route discovery is an overkill, thus favoring MAC-layer salvaging. However, if a communication attempt fails due to a permanent problem such as node mobility, MAC-layer salvaging may be able to save the current packet but not the next one because the receiver moves farther away from the sender. Now, network-layer salvaging is invoked by saving the packet at hand as well as those in transit along the routing path. In other words, they play roles in different areas and improve the packet-delivery capability synergistically if both of them are employed.

\subsection{Enhancing Spatial Reusability}

This subsection overviews three mechanisms that enhance the spatial reusability: enhanced carrier sensing (ECS), transmit power control (TPC), and transmit rate control (TRC).

Recently, researchers have proposed enhanced carrier sensing (ECS) schemes that speculate on the outcome of a transmission based on signal strength and communication distance. If the speculation tells that the transmission would not cause any problem, packets are transmitted even if the transmitter senses the carrier above the predefined threshold. For instance, $\mathrm{Xu}$ et al. proposed a Conservative Clear-to-Send Reply (CCR) scheme [27] in which a node replies only for a Request-to-Send (RTS) when the receiving power of the RTS is higher than a certain threshold, ensuring that the sender is in the proximity of the receiver. Ye et al. proposed an aggressive virtual carrier sensing (AVCS) scheme [33] that allows a node to start its communication, which is prohibited by the virtual CS using the RTS-CTS handshake when the communication distance is short. However, these schemes have a limited practical value because most of the routing algorithms developed for wireless ad hoc networks offer shortest paths for a given source-destination pair and, thus, the physical distance for each hop is usually in the order of maximum transmit range supported by the radio hardware. More recently, a more direct approach has been proposed. Location Enhanced DCF (LED) [34] and Adaptive Physical Carrier Sensing (APCS) [35] change the carrier sense threshold when it is beneficial. Therefore, the decision whether a certain interference level is a carrier signal or a noise is not deterministic anymore but a function of the radio environment in the proximity.

Another way to enhance the spatial reusability is TPC. It allows a node to adjust and optimize its radio transmit power to reach the receiver node, but not more than that. A key benefit of the TPC schemes is energy conservation, but it also reduces interference, allowing more concurrent data transfers. However, most TPC-based protocols [36], [37] are concerned primarily with low power transmission of data packets and assume that control packets are transmitted at the highest radio power. Therefore, they do not directly increase the spatial reusability of the spectral resource. On the contrary, Smallest Common Power (COMPOW) [37] and Power-Stepped Protocol (PSP) [19] use the same radio power for both data and control packets, but they incur an additional overhead to compute the optimal transmit power level.

Alternatively, TRC exploits a physical-layer multirate capability to make a data transfer more robust to interference. Even if the SIR is not high, a certain data rate is always achievable based on Shannon's theorem, which can be exploited to improve the network performance. For example, a receiver measures the channel quality based on the RTS it received and then informs the appropriate transmit rate to the sender so that the channel can always be utilized at the highest feasible data rate. Shepard showed the theoretic bounds of the network throughput, assuming that the transmit rate is arbitrarily adjustable [24]. Prabhakar et al. proposed an energy-efficient communication schedule that takes the TRC capability into account [38] and Sadeghi et al. proposed an opportunistic media access scheme that better exploits the channel via TRC and channel quality information [39]. More recently, Yang and Vaidya showed via analysis and simulation that TRC can significantly improve the overall network throughput jointly with carrier sensing control [26]. The ECS, TRC, and TPC schemes can be integrated with the proposed MASA algorithm when the corresponding hardware capability is available.

\section{Conclusion and Future Work}

Carrier sensing MAC protocols avoid collisions by employing aggressive carrier sensing, but it makes them unable to maximize the spatial spectral utilization. This paper analyzes the upper bound throughput of a carrier sensing MAC and observes that the network throughput can be greatly improved if the capture effect is taken into consideration.

The proposed MASA algorithm adopts a fixed, small carrier sense range but adaptively adjusts the communication distance via salvaging packets. While the former increases spatial reusability, the latter alleviates the collision problem. For practicality, we considered implementation of MASA based on the DCF specification. Our extensive simulation study showed that MASA enhances the network performance regardless of mobility, traffic intensity, and the routing algorithm used. In particular, it reduces packet delay significantly.

The MASA algorithm is considered the most preferable in a wireless ad hoc network where a large number of nodes exchange small packets, which is typically the case in wireless sensor networks. The application of MASA in this area is our future work. How to elect a salvager deterministically rather than randomly between each pair of communicating nodes is also part of our future work. 


\section{RefERENCES}

[1] C. Yu, K.G. Shin, and L. Song, "Link-Layer Salvaging for Making Progress in Mobile Ad Hoc Networks," Proc. ACM MobiHoc, 2005.

[2] B. Bertsekas and R. Gallager, "Multiaccess Communication," Data Networks, chapter 4, second ed., Prentice Hall, 1992.

[3] A. Kamerman and L. Monteban, "WaveLAN-II: A High-Performance Wireless LAN for the Unlicensed Band," Bell Labs Technical J., pp. 118-133, Summer 1997.

[4] M. Grossglauser and D. Tse, "Mobility Increases the Capacity of Ad-Hoc Wireless Networks," Proc. IEEE INFOCOM, 2001.

[5] M. Zorzi and R. Rao, "Capture and Retransmission Control in Mobile Radio," IEEE J. Selected Areas in Comm., vol. 12, no. 8, pp. 1289-1298, 1994.

[6] D.B. Johnson and D. Maltz, "Dynamic Source Routing in Ad-Hoc Wireless Networks," Mobile Computing, T. Imielinski and H. Korth, eds., chapter 5, pp. 153-181, Kluwer Academic, 1996.

[7] C.E. Perkins and E. Royer, "Ad-Hoc On-Demand Distance Vector Routing," Proc. IEEE Workshop Mobile Computing Systems and Applications, pp. 90-100, 1999.

[8] S. Biswas and R. Morris, "Opportunistic Routing in Multi-Hop Wireless Networks," Proc. Second Workshop Hot Topics in Networks (HotNets-II), 2003.

[9] B. Blum, T. He, S. Son, and J. Stankovic, "IGF: A State-Free Robust Communication Protocol for Wireless Sensor Networks," Technical Report CS-2003-11, Univ. of Virginia, Apr. 2003.

[10] T. He, J.A. Stankovic, C. Lu, and T.F. Abdelzaher, "SPEED: A Stateless Protocol for Real-Time Communication in Sensor Networks," Proc. IEEE Int'l Conf. Distributed Computing Systems (ICDCS '03), May 2003.

[11] P. Skraba, H. Aghajan, and A. Bahai, "Distributed Passive Routing Decisions in Mobile Ad-Hoc Networks," Proc. IEEE Vehicular Technology Conf. (VTC '04), Sept. 2004.

[12] M. Zorzi and R. Rao, "Geographic Random Forwarding (GeRaF) for Ad Hoc and Sensor Networks: Energy and Latency Performance," IEEE Trans. Mobile Computing, vol. 2, no. 4, Oct.-Dec. 2003.

[13] J. Bicket, D. Aguayo, S. Biswas, and R. Morris, "Architecture and Evaluation of an Unplanned 802.11b Mesh Network," Proc. IEEE/ ACM MobiCom, 2005.

[14] D.S.J. De Couto, D. Aguayo, B.A. Chambers, and R. Morris, "Performance of Multihop Wireless Networks: Shortest Path Is Not Enough," Proc. First Workshop Hot Topics in Networks (HotNets-I), 2002.

[15] R. Draves, J. Padhye, and B. Zill, "Comparison of Routing Metrics for Static Multi-Hop Wireless Networks," Proc. ACM SIGCOMM, 2004.

[16] "ns-2 Network Simulator," http://www.isi.edu/nsnam/ns/, April 2005

[17] A. Bonsall, D. Wang, and D.D. Stancil, "Propagation Model Embedded in a Wireless Network Simulator," Proc. Third Ann. Wireless Comm. Conf., 1998.

[18] B. Tuch, "Development of WaveLAN, An ISM Wireless LAN," ATET Technical J., vol. 72, no. 4, pp. 27-37, July/Aug. 1993.

[19] C. Yu, K.G. Shin, and B. Lee, "Power-Stepped Protocol: Enhancing Spatial Utilization in a Clustered Mobile Ad Hoc Network," IEEE J. Selected Areas in Comm. (J-SAC '04), vol. 22, no. 7, pp. 1322-1334, Sept. 2004.

[20] L. Kleinrock and F.A. Tabagi, "Packet Switching in Radio Channels: Part I-Carrier Sense Multiple-Access Models and Their Throughput-Delay Characteristics," IEEE Trans. Comm., vol. 23, no. 12, Dec. 1975.

[21] Local and Metropolitan Area Network, Part 11: Wireless LAN Medium Access Control and Physical Layer Specifications, IEEE Std 802.111999, http://standards.ieee.org/getieee802/download/802.111999.pdf, 1999.

[22] K. Pahlavan and P. Krishnamurthy, "Network Planning," Principles of Wireless Networks, chapter 5, Prentice Hall, 2002.

[23] P. Gupta and P.R. Kumar, "The Capacity of Wireless Networks," IEEE Trans. Information Theory, vol. 46, no. 2, pp. 388-404, Mar. 2000.

[24] T.J. Shepard, "A Channel Access Scheme for Large Dense Packet Radio Networks," Proc. ACM SIGCOMM, 1996.

[25] J. Zhu, X. Guo, L.L. Yang, and W.S. Conner, "Leveraging Spatial Reuse in 802.11 Mesh Networks with Enhanced Physical Carrier Sensing," Proc. IEEE Int'l Conf. Comm. (ICC '04), 2004.

[26] X. Yang and N. Vaidya, "On the Physical Carrier Sense in Wireless Ad Hoc Networks," Proc. IEEE INFOCOM, 2005.
[27] K. Xu, M. Gerla, and S. Bae, "Effectiveness of RTS/CTS Handshake in IEEE 802.11 Based Ad Hoc Networks," Ad Hoc Network J., vol. 1, no. 1, pp. 107-123, July 2003.

[28] L.E. Miller, "Carrier Sense Threshold/Range Control: Compilation of MANET Email Messages," http://www.antd.nist.gov/ wctg/manet/docs/carriersense.pdf, Jan. 2004.

[29] P. Mühlethaler and A. Najid, "Throughput Optimization in Multihop CSMA Mobile Adhoc Networks," Proc. European Wireless Conf., 2004.

[30] S. Xu and T. Saadawi, "Does the IEEE 802.11 MAC Protocol Work Well in Multihop Wireless Ad Hoc Networks?" IEEE Comm. Magazine, pp. 130-137, June 2001.

[31] A. Valera, W. Seah, and S.V. Rao, "Cooperative Packet Caching and Shortest Multipath Routing in Mobile Ad Hoc Networks," Proc. IEEE INFOCOM, 2003.

[32] B. Karp, "Geographic Routing for Wireless Networks," PhD dissertation, Harvard Univ., Oct. 2000.

[33] F. Ye, S. Yi, and B. Sikdar, "Improving Spatial Reuse of IEEE 802.11 Based Ad Hoc Networks," Proc. IEEE GLOBECOM, 2003.

[34] T. Nadeem, L. Ji, A. Agrawala, and J. Agre, "Location Enhancement to IEEE 802.11 DCF," Proc. IEEE INFOCOM, 2005.

[35] J. Zhu, X. Guo, L.L. Yang, W.S. Conner, S. Roy, and M.H. Hazra, "Adapting Physical Carrier Sensing to Maximize Spatial Reuse in 802.11 Mesh Networks," Wireless Comm. and Mobile Computing, vol. 4, no. 8, pp. 933-946, Dec. 2004.

[36] E.-S. Jung and N.H. Vaidya, "A Power Control MAC Protocol for Ad Hoc Networks," Proc. IEEE/ACM MobiCom, 2002.

[37] S. Narayanaswamy, V. Kawadia, R.S. Sreenivas, and P.R. Kumar, "Power Control in Ad-Hoc Networks: Theory, Architecture, Algorithm and Implementation of the COMPOW Protocol," Proc. European Wireless Conf., pp. 156-162, 2002.

[38] B. Prabhakar, E. Uysal-Biyikoglu, and A. El Gamal, "EnergyEfficient Transmission over a Wireless Link via Lazy Packet Scheduling," Proc. IEEE INFOCOM, pp. 386-394, 2001.

[39] B. Sadeghi, V. Kanodia, A. Sabharwal, and E. Knightly, “Opportunistic Media Access for Multirate Ad Hoc Networks," Proc. IEEE/ACM MobiCom, 2002. 